\title{
Correlation between histological type of epidermoid carcinoma in the head and neck and the presence of HPV assessed by PI6 imunoexpression
}

\begin{abstract}
Introduction: Head and Neck Squamous Cell Carcinoma (HNSCC) is a malignant disease entity with a high prevalence in the world population. Among its major risk factors, there is a persistent infection with Human Papilloma Virus (HPV), which has been related to a better prognosis in patients. HPV infection results in an immunoreactivity of p16 protein that has been used as a marker of the oncogenic lineage by this etiologic agent. The lack of national case series relating the HNSCC with HPV and p16 encouraged us to develop this research.
\end{abstract}

Objective: To analyze epidemiological aspects of patients affected by HNSCC (age, sex and location of the lesion), and relate them to the prevalence of HPV infection. To evaluate the presence of virus stigmas in the samples (koilocytes). To correlate histological types and differentiation of HNSCC positive for p16.

Methods: We have analyzed 51 cases of HNSCC diagnosed between January 2005 and December 2015, seeking epidemiological profile of patients, and being the samples subjected to histopathology and immunohistochemistry for $\mathrm{p} 16$.

Results: Of the 51 cases evaluated, 42 were males and 9 females, mean age of 61 years. There was a higher percentage of tumors affecting the larynx (43\%), with higher prevalence of keratinized cancers on non keratinized. Koilocytosis was observed in $56.9 \%$ of cases, and immunostaining for p16 was $49.02 \%$, predominantly in tumors not keratinized $(\mathrm{p}=0.03532)$.

Conclusion: The present study has demonstrated that the infection prevalence of HPV in HNSCC, through the immunostaining with p16, was present in $49.02 \%$ of cases. Toward the epidemiological profile, carcinomas were more common in male individuals with middle ages of 61 years and in the larynx as more often topography. Koilocytosis was found in 29 cases, corresponding to $56.86 \%$ of our sample. The immunoreactivity of p16 protein predominated in non-keratinized tumors.

Keywords: head and neck carcinoma, human papilloma virus, p16
Volume 10 Issue $6-2019$

\author{
Marcos Paulo R Sanches,' Luis Martins \\ Collaço,' Johnny Godoy,' Daniele Alves² \\ 'Evangelical College of Paraná, Curitiba, Paraná, Brazil \\ ${ }^{2}$ Pontificial Catholic University of Paraná, Brazil
}

Correspondence: Marcos Paulo R Sanches, Evangelical College of Paraná, Curitiba, Paraná, Brazil, Email mprsanche@hotmail.com

Received: October 25, 2019 | Published: November 19, 2019
Abbreviations: HNSCC, head and neck squamous cell carcinoma; HPV, human papilloma virus; SCC, squamous cell carcinoma; INCA, National Cancer Institute José Alencar Gomes da Silva; NQ, non-keratinized; PCR, polymerase chain reaction; IHQ, immune-histochemistry; RBP, retinoblastoma protein; TMAs, tissue micro arrays

\section{Introduction}

Head and Neck Cancer is a broad term used to describe a diverse group of malignancies, originated in the upper aero-digestive tract, including the oral and tongue cavity, pharynx, larynx, salivary glands, ear, nasal cavity and paranasal sinuses. Among its main representatives: the squamous cell carcinoma (SCC), described as an aggressive epithelial malignant disease that accounts for $95 \%$ of tumors in these locations, being more common in the mouth. ${ }^{1}$ This specific species of tumor is so significant that, in 2012, it was estimated that the SCC of the tongue, oral cavity, pharynx and larynx together totaled in 686,300 new cases and 375,700 cancer deaths worldwide. ${ }^{2}$ And, according to data from the National Cancer Institute José Alencar Gomes da Silva (INCA), in 2016 there were 15,490 cases of oral SCC in Brazil, 11,140 in men and 4,350 affecting women.

Regarding their classification, head and neck SCC (SCCPS) are subdivided into Non-Keratinized (NQ), Keratinized (K) and Hybrid
$(\mathrm{H}){ }^{3,4}$ The non-keratinized form is the one with the best prognosis and, when found in its basaloid differentiation, the classic form of carcinoma presentation. ${ }^{3,4}$

HPV was defined as a risk factor for cervical cancer in the 1970s; being responsible for more than $90 \%$ of malignant neoplasm of this organ nowadays. However, it has still been separately associated with oral and oropharyngeal squamous cell carcinoma, especially regarded to palatine tonsil involvement. ${ }^{5}$ The incidence of HPVpositive CECCP worldwide ranges from 20 to $80 \%$, with the majority of incidence occurring in patients aged 40 to 55 years, without risk factors and linked to persistent high-risk HPV infection. ${ }^{6}$

This new type of HPV-positive tumor develops mainly in the palatine tonsils and tongue and manifests different epidemiological, molecular and clinical characteristics than classic CECCP. Among the main risk factors there is prognosis for white male individuals whose number of partners is high and engage in oral sex, and bearers of genital warts. ${ }^{7}$ In addition, it was discovered that the natural history of development of this disease shows a better prognosis compared to cases of patients with HPV-negative tumors. ${ }^{8}$

The incidence of HPV-infected malignancies has been increasing, as shown by Attner et al., ${ }^{9}$ providing us with evidence to support the considerable increase in HPV-positive tongue-based cancers from 
1998 to 2007. Therefore, Virus detection methods in head and neck carcinomas have risen in numbers because, aside from instructing the nature of tumors, this identification also helps to assess patient prognosis. ${ }^{10}$

HPV is a double-stranded DNA virus which family spans over 170 subtypes. ${ }^{11}$ It presents tropism by skin and mucosal epithelial cells, and its replication occurs in cell nuclei (Martinez, Avila, Caballero, 2012). After infectation, the main cytopathic effect of the virus is the development of koilocytosis, which is defined by increased concentration of koilocytes, a specific cell type and pathognomonic to HPV infection. (Silva, 2010). This element is a superficial cell with cytoplasmic cavitation and nuclear atypia. ${ }^{12}$ HPV DNA molecular detection is the gold standard method for virus identification and can be performed by hybrid capture and polymerase chain reaction (PCR), FISH, or immune-histochemistry (IHQ) for protein $\mathrm{p} 16 .{ }^{2,6,10,11}$ This latter method is emphasized in the present study because, while p16 biomarkeration in HPV negative CECCP is extremely rare, the positivity of this same protein in virus-infected lesions has been frequent. In addition, the positivity of p16 biomarker in head and neck squamous cell carcinomas has been proposed as a reliable and reproducible prognostic marker. ${ }^{13}$ Cyclins are proteins related to the promotion of cell growth and are expressed in the middle and end of the G1 phase. Over expression results in increased cell proliferation, and in carcinomas, increased expression is related to aggressiveness, infiltration and metastasis (Suresh, 2015).

In regard to molecular biology, high-risk carcinogenic HPV continuously and irregularly expresses oncoproteins E6 and E7. This anomalous production results in cell immortalization and genomic instability, which events may lead to the progression of cancerinfected tissue. ${ }^{14}$ The significant increase in E7 oncoprotein by functionally inactivating the retinoblastoma protein (RBP) triggers the increase of p16 expression, which has a diagnostic relevance because it is detected by the immuno-histochemistry technique. ${ }^{15}$ The increase in E6, in turn, has the role of complementing the action of E7 by containing the pro-apoptotic cellular response by promoting unscheduled DNA replication and encouraging cell proliferation. ${ }^{16}$

It is known that there are national studies that correlate histological type of head and neck neoplasms with HPV, and few studies correlate their viral stigmas (koilocytes) with p16 biomarkeration. Furthermore, it is a known fact that HPV positivity is considered to be an independent and strong prognostic factor for CPEC. Therefore, studies such as the present study are necessary to confirm the prevalence of HPV-positive cases of CPEC, and to prove its relationship with $\mathrm{p} 16$ protein labeling.

\section{The main goal}

To evaluate the prevalence of HPV infection in cases of Head and Neck Squamous Cell Carcinoma, through P16 positive.

\section{Specific goals}

a. Promote descriptive analysis of epidemiological data, such as age, gender, and lesion location.

b. Evaluate, under optical microscopy, the presence of viral stigmas in the samples (koilocytes).

c. Correlate the histological types and differentiation of CECCP with 16 positivity.

\section{Methodology}

The research was performed in a cross-sectional and retrospective manner in the electronic archives of the Curitiba Pathology Center
(Nossa Senhora das Gracias Hospital, Curitiba, Paraná) and the Hospital Universitário Evangélico from Curitiba, searching for all cases with anatomopathological diagnosis of head and neck SCC, having occurred from January 2005 to December 2015. The project was approved by the Research Ethics Committee of the Evangelical Society of Paraná - CEP/SEB, under opinion number 1.453.251.

From the selected patients, data related to gender, age and topography of the lesion were compiled from the medical records. From the archives, paraffin blocks and histopathological slides stained by the Hematoxylin and Eosin (HE) technique were obtained, both containing samples previously diagnosed with CECCP. Exclusion criteria were incomplete medical records, cases in which the slides and blocks selected by the electronic medical record were not found, as well as those that, after mounting the TMA, showed no neoplasia.

The histopathological slides were revised under multi-head microscopes in order to classify the histological type of the lesion, its differentiation, and also to verify the presence or absence of characteristic HPV (koilocyte) histological stigmas. In HE, CECCP were cataloged as "Keratinized", when they presented corneal pearl and individual keratinization; "Non-Keratinized" when they did not show signs of keratin production; and "Mixed", when the areas that had both characteristics were present. Identification of koilocytes was characterized by cells of the intermediate or superficial stratum of the epithelium, with well-marked cytoplasm clear halo and nuclear atypia (Figure 1).

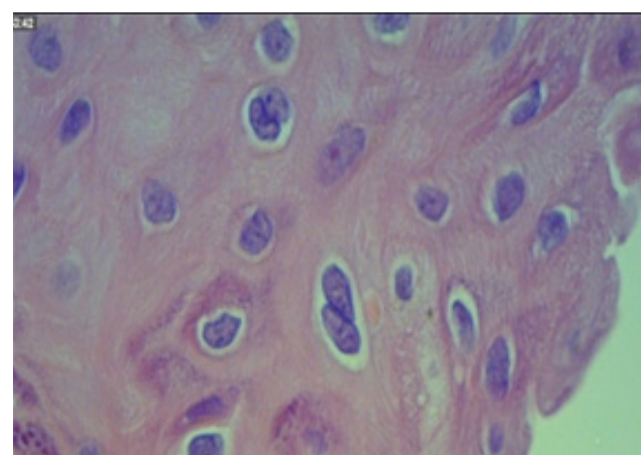

Figure I Coilocyte in squamous neoplasia.

Note: Photomicrography demonstrating koilocyte (arrow) in squamous neoplasia. Hematoxylin-Eosin 400x magnification.

After selecting the histopathological blocks and slides containing the carcinoma samples, the cases were reviewed in order to select the sample areas for the making of the multi-sample tissue blocks (or TMAs=tissuemicroarrays). Following the preparation of the multi-sample blocks (TMAs), these were used to prepare the multisample slides, in which the p16 biomarker was tested by immunehistochemistry.

Deriving from the histological slides referring to the study cases, the representative sample regions of the lesion were selected and duly marked with a rear projection pen. Through the mirror system, the marked blade is used to locate the same region in the donor block. The technique of multisampling blocks or receptors (TMAs) consists of drilling the donor block in the sample area previously marked with the consequent obtention of a tissue cylinder inside the needle and; thereafter, implantation of this tissue into the receptor block. Each sample fragment taken from the donor block was placed in the recipient block according to the "Cartesian plane" map - the columns are identified with letters and the rows with numbers (Figure 2). 


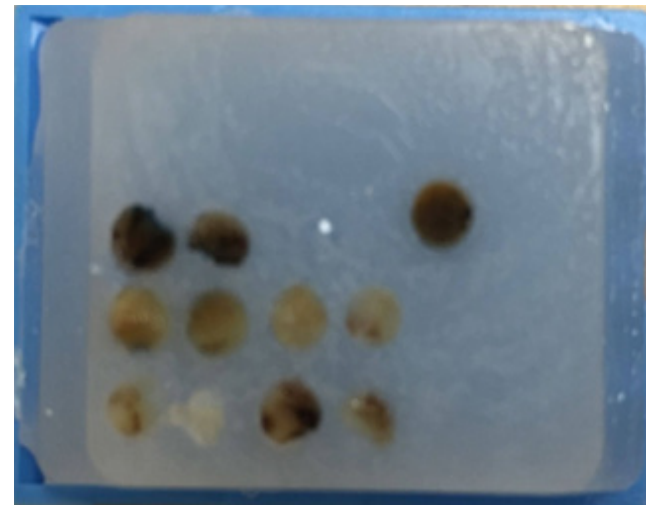

Figure 2 Multi-sample block slide.

The histological slides from the artisanal TMA blocks were submitted to the immune-histochemistry process, in which the study of p16 protein (FLEX TRS HIGH, Dako $\left.{ }^{\circledR}\right)$ was established. This latter process was divided into two distinct phases: first, the application of the standard immune-histochemistry technique to the material through the AutostainerLink 48 automatic staining machine, Dako ${ }^{\circledR}$. Subsequently, reading of the area with tissue immunostaining with the Olympus ${ }^{\circledR}$ BX 50 multi-head optical microscope was considered positive when the cases were strong and diffuse brownbrown cytoplasmic and nuclear marking (Figure 3) (Figure 4).

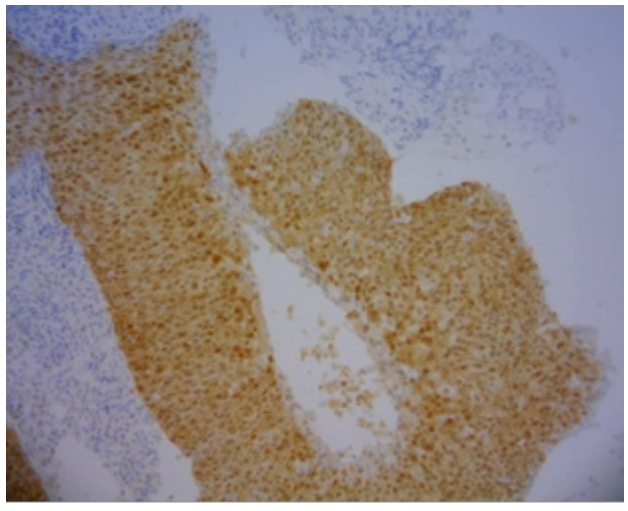

Figure 3 Positive for PI6.

Note: Photomicrograph of pl6 positive squamous cell carcinoma (brown area). 100x Zoom.

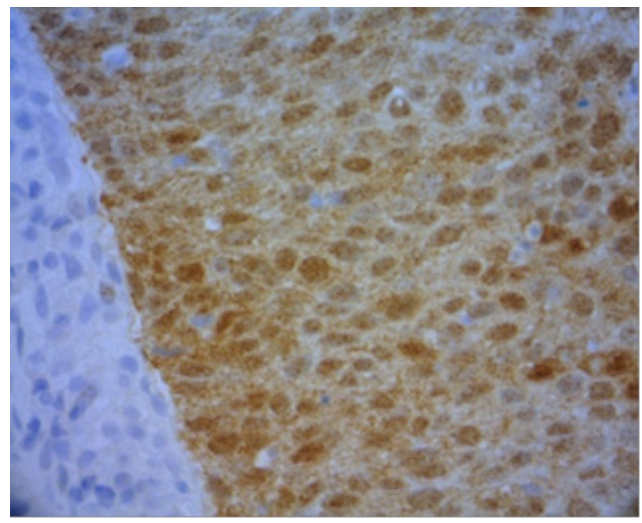

Figure 4 Details from positive pl6.

Note: Detail of pl6 positivity in squamous cell carcinoma demonstrating strong cytoplasmic and nuclear labeling (brown). 400x Zoom.
All results and information obtained were labeled according to data protocol, and then expressed through graphs and tables, developed from Excel spreadsheets. In this study, the chi-square test was used to analyze the difference between the observed result and the expected result. However, this test is limited. When more than $1 / 4$ of the expected values in a $2 \times 2$ table were less than 5 , we used Fisher's exact test and Cramer's V statistic. For tables with variables that had more than two parameters, larger than $2 \times 2$, we used logistic regression for dependency analysis.

All the material containing the information was the responsibility of the researchers themselves, ensuring the maintenance of confidentiality and confidentiality.

\section{Results}

The present sample consisted of 51 cases, 42 males and 9 females, with ages ranging from 44 to 89 years, average of 61 years and standard deviation of 10.64 years. The most frequent location of the tumors was in the larynx territory, with 22 cases, followed by tongue lesions, 16 cases, 10 in the mouth and 3 in palatine tonsils.

In regard of results in our research, head and neck SCC prevailed in males, which accounted for 42 cases $(82.35 \%)$. In this group, the most prevalent topography was the larynx, with 19 cases $(45 \%)$. Tongue tumors accounted for 13 cases (31\%), mouth $8(19 \%)$ and tonsils, $2(5 \%)$. On the other hand, in females, there were the same amount of laryngeal and tongue tumors, 3 cases each (33\%), two in the mouth (22\%) and a single case in tonsils (11\%) (Figure 5).

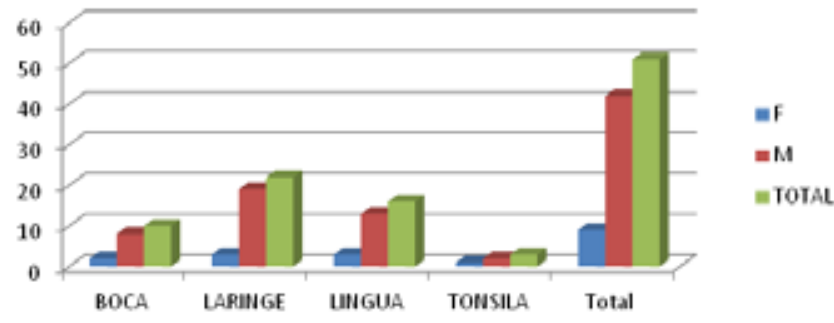

Figure 5 Relation between sex and topography of injury.

Regarding the histological differentiation pattern, most of the lesions in the sample were keratinized, as shown in Table 1.

Table I Proportion between histological types and injury topography

\begin{tabular}{llllll}
\hline $\begin{array}{l}\text { Line } \\
\text { labels }\end{array}$ & Mixed & $\begin{array}{l}\text { Non- } \\
\text { keratinized }\end{array}$ & Keratinized & $\begin{array}{l}\text { Grand } \\
\text { total }\end{array}$ & $\%$ \\
\hline Mouth & 1 & 3 & 6 & 10 & $20 \%$ \\
Larynx & 6 & 6 & 10 & 22 & $43 \%$ \\
Tongue & 3 & 5 & 8 & 16 & $31 \%$ \\
$\begin{array}{l}\text { Tonsils } \\
\text { Grand }\end{array}$ & 10 & 17 & 24 & 3 & $6 \%$ \\
Total & & & & 51 & $100 \%$ \\
\hline
\end{tabular}

Among men, the keratinized tumors were 21 (50\%), and among the non-keratinized and mixed tumors there was some homogeneity, corresponding to 11 and 10 cases, respectively. Among women, no cases of mixed tumors were found, and the highest prevalence was non-keratinized tumors, making up 66.67\% (Table 2). 
Table 2 Distribution of prevalence of histological type of injury and sex

\begin{tabular}{llllll}
\hline & Female & $\%$ & Male & $\%$ & Total \\
\hline Mixed & 0 & 0 & 10 & $24 \%$ & 10 \\
Non-keratinized & 6 & $66,67 \%$ & 11 & $26 \%$ & 17 \\
Keratinized & 3 & $33,33 \%$ & 21 & $50 \%$ & 24 \\
Grand Total & 9 & $100 \%$ & 42 & $100 \%$ & 51 \\
\hline
\end{tabular}

Tumors displaying koilocyte expression predominated in our sample, $29(56.86 \%)$ of 51 cases. In this group of tumors with koilocytosis, the predominance was in laryngeal SCC, with $45 \%$, followed by tongue tumors in which the diagnostic cell was present in $34 \%$ of the lesions (Table 3). There is no correlation between these variables at the $5 \%$ level. The logistic regression test was used for this calculation; the calculated p-value was 0.329 .

Table 3 Relationship between coilocytosis and topography of injuries

\begin{tabular}{llllll}
\hline & No & $\%$ & Yes & $\%$ & Total \\
\hline Mouth & 5 & $23 \%$ & 5 & $17 \%$ & 10 \\
Larynx & 9 & $41 \%$ & 13 & $45 \%$ & 22 \\
Tongue & 6 & $27 \%$ & 10 & $34 \%$ & 16 \\
Tosils & 2 & $9 \%$ & 1 & $3 \%$ & 3 \\
Grand Total & 22 & $100 \%$ & 29 & $100 \%$ & 51 \\
\hline
\end{tabular}

The prevalence of koilocytes occurred in keratinized tumors, with an index of $72 \%$, as shown in Table 4 .

Table 4 Relation between coilocytosis and histological type of injuries

\begin{tabular}{llllll}
\hline Rótulos de linha & No & $\%$ & Yes & $\%$ & Grand total \\
\hline Mixed & 4 & $18 \%$ & 6 & $21 \%$ & 10 \\
Non-keratinized & 15 & $68 \%$ & 2 & $7 \%$ & 17 \\
Keratinized & 3 & $14 \%$ & 21 & $72 \%$ & 24 \\
Grand Total & 22 & $100 \%$ & 29 & $100 \%$ & 51
\end{tabular}

Correlating topography with positivity for $\mathrm{p} 16$, the present sample showed no statistically significant relationship $(\mathrm{p}=0.7821)$ (Table 5).

Table 5 Relation between pl 6 biomarking and injury topography

\begin{tabular}{llllll}
\hline & Mouth & Larynx & Tongue & Tonsils & Total \\
\hline Negative & 6 & 12 & 7 & 1 & 26 \\
Positive & 4 & 10 & 9 & 2 & 25 \\
Total & 10 & 22 & 16 & 3 & 51 \\
\hline
\end{tabular}

The prevalence of p16 positivity among all lesions was $49.02 \%$ (25 cases). Comparing p16 expression and histological type, the highest positivity was in non-keratinized tumors, making up 12 cases $(48 \%)$. There was statistical significance by Fisher's exact test for this comparison $(\mathrm{p}=0.03532)$ (Table 6).

Table 6 Relationship between p 16 biomarking and histological type of injuries

\begin{tabular}{llllll}
\hline & Negative & $\%$ & Positive & $\%$ & Total \\
\hline Mixed & 8 & $31 \%$ & 2 & $8 \%$ & 10 \\
Non-keratinized & 5 & $19 \%$ & 12 & $48 \%$ & 17 \\
Keratinized & 13 & $50 \%$ & 11 & $44 \%$ & 24 \\
Grand Total & 26 & $100 \%$ & 25 & $100 \%$ & 51 \\
\hline
\end{tabular}

Relating the presence of koilocytes and positivity for p16 immunoexpression was positive in 25 cases (49\%) (Table 7).

Table 7 Relationship between coilocytosis and biomarking of pl6

\begin{tabular}{lllll}
\hline Line labels & No & Yes & Grand total & $\%$ \\
\hline Negative & 8 & 18 & 26 & $51 \%$ \\
Positive & 14 & 11 & 25 & $49 \%$ \\
Grand Total & 22 & 29 & 51 & $100 \%$ \\
\hline
\end{tabular}

\section{Discussion}

During the research fifty-one cases of head and neck squamous cell carcinoma were studied in the present series, analyzing the epidemiological profile of the patients and the positivity for p16 protein immunostaining.

Over the recent years, numerous immunohistochemical markers have been studied in the management of oral cavity squamous cell carcinomas, as well as in HPV research in this cancers. ${ }^{10}$ The p16 protein, which acts as a tumor suppressor protein by competitively inhibiting cyclin-dependent kinesis, is an important example of this new diagnostic strain. It is found in low concentrations in the normal epithelium, and its expression is increased in proliferative and inflammatory processes (Britto, Paula and Sadi, 2014). And the main reason for its study, allowing the analysis of viral infection in these tumor types, is the fact that HPV positive patients have shown a better prognosis, ${ }^{10}$ as well as a better response to treatment and sensitivity to radiotherapy. ${ }^{17}$

In the present research, there was a prevalence of head and neck CPB in male patients, with 42 out of 51 patients $(82.35 \%)$, with a mean age of 61 years, which corroborates the data released by INCA in 2016, in which this index was $71.92 \%$. In addition, this higher prevalence in men is also found in the research conducted by Antonsson ${ }^{17}$ in Queensland, Australia, which showed a higher number of these tumors in men (74\%) with a mean age of 62 years.

Regarding the topography of the lesion, there is a higher concentration of SCC in the books, making a total of $95 \%$ for head and neck tumors. ${ }^{1}$ It is also noticeable in the Australian study by Antonsson, ${ }^{17}$ which demonstrates tumor involvement of the oral cavity in $36 \%$ of tumors. However, in our study, the lesions showed higher prevalence in another location, in the larynx, corresponding to $43 \%$ of cases. Possibly, this discrepancy may be related to a different anatomical division performed by the Australian study, which subdivides a larger number of compartments. While our sample was grouped into mouth, tongue, tonsil and larynx, the study sample is classified into mouth, oropharynx, hypopharynx and larynx.

In the present study, it was observed that the cases of keratinized head and neck squamous cell carcinoma are the most prevalent $(47.06 \%)$, except for women, where a predominance of the pattern of non-keratinized tumors was observed. Regarding this factor, no comparative parameters were found in the literature.

Koilocytosis, which is the morphological expression of HPV infection, which can be noted in cytopathology and histopathology, was evidenced in 29 cases $(56.86 \%)$ of this sample, predominantly in keratinized tumors. Regarding this factor, no comparative parameters were found in the referential literature.

An Immunoexpression of $\mathrm{p} 16$ protein, which is a biological marker related to neoplastic induction by HPV, showed a prevalence of $49.02 \%$ in the present series, predominating in non-keratinized tumors 
and laryngeal localization (19.60\%). Regarding this immunostaining, Fischer et al. $^{8}$ described that among 365 CECCP biopsies, p16 expression was present in 288 cases $(78.9 \%)$, with $32.05 \%$ found in the mouth topography. Quite possibly, this discrepancy from the values found in the literature and our study may be related to the sample size, and to a higher concentration of cases in the mouth and oropharynx topography, which are the most prevalent places in the literature. ${ }^{18-25}$

Thus, the present study term-paper aims to contribute to a better understanding of the oncogenesis of head and neck squamous cell carcinomas. Notwithstanding its results, it would be interesting to increase the number of cases for greater representativeness of this group of lesions, as well as to enable other studies to compare, for example, the biological behavior and growth of these tumors with their prognosis. ${ }^{26-33}$

\section{Conclusion}

The present term-paper study concludes:

i. The prevalence of HPV infection in head and neck SCC by p16 immunostaining was $49.02 \%$.

ii. Regarding the epidemiological profile, SCCs predominated in males, with an average age of 61 years, and with the most frequent topography as the larynx.

iii. The koilocytic expression was found in 29 cases, corresponding to $56.86 \%$ of our sample.

iv. Immunoexpression of $\mathrm{p} 16$ protein predominated in cases of nonkeratinized tumors $(\mathrm{p}=0.03532)$

\section{Acknowledgments}

None.

\section{Funding}

None.

\section{Conflicts of interest}

The author and co-authors have no conflicts of interest relevant to this article.

\section{References}

1. Lingen MW. Head and neck. In: Kumar V, Abbas AK, Fausto N, editors. Robbins and cotran pathology: pathological bases of diseases. 8th ed. Rio de Janeiro: Elsevier; 2008

2. Vermorken JB. Treatment of elderly patients with squamous cell carcinoma of the head and neck. Front Oncol. 2016;6:199.

3. Cooper T, Biron V, Adam B, et al. Prognostic utility of basaloid differentiation in oropharyngeal cancer. J Otolaryngol Head Neck Surg. 2013;42:57.

4. El-Mofty SK. HPV-related squamous cell carcinoma variants in the head and neck. Head Neck Pathol. 2012;6 Suppl 1:S55-S62.

5. Melo AUC, Ribeiro CF, Santos TS, et al. Human papillomavirus as a risk factor for oral and oropharyngeal carcinoma. Rev Bras Cir Cabeça Pescoço. 2012;41(4):207-211.

6. Alves AVF, Ribeiro DR, Lima SO, et al. Expression of Ki-67 and P16 INK4a in chemically-induced perioral squamous cell carcinomas in mice. Rev Col Bras Cir. 2016;43(2):72-799.

7. Antonsson A, Neale RE, Boros S, et al. Human pappilomavirus status and p16INK4A expressions in patients with mucosal esquamous cell carcinoma of the head and neck in Queensland, Australia. Cancer Epidemiol. 2015;39(2):174-181.

8. Attner P, Näsman A, Du J, et al. Survival in patients with human papillomavirus positive tonsillar cancer in relation to treatment. Int $J$ Cancer. 2012;131(5):1124-1130.

9. Chernock RD, El-Mofty SK, Thorstad WL et al. HPV-related nonkeratinizing squamous cell carcinoma of the oropharynx: Utility of microscopic features in predicting patient outcome. Head Neck Pathol. 2009;3(3):186-194.

10. Dayyani F, Etzel CJ, Liu M, et al. Meta-analysis of the impact of human papillomavirus (HPV) on cancer risk and overall survival in head and neck squamous cell carcinomas (HNSCC). Head Neck Oncol. 2010;2:15.

11. Fischer CA, Zlobec I, Green E, et al. Is the improved prognosis of $\mathrm{p} 16$ positive oropharyngeal squamous cell carcinoma dependent of the treatment modality. Int J Cancer. 2010;126(5):1256-1262.

12. Freitas LB, Chen Z, Muqui EF, et al. Human papillomavirus 16 nonEuropean variants are preferentially associated with high-grade cervical lesions. PLoS One. 2014;9(7):e100746.

13. Ganti K, Broniarczyk J, Manoubi W, et al. The human papillomavirus E6 PDZ binding motif: from life cycle to malignancy. Viruses. 2015;7(7):3530-3551.

14. Gasche JA, GoelA. Epigenetic mechanisms in oral carcinogenesis. Future Oncol. 2012;8(11):1407-1425.

15. Gasche JA, Hoffmann J, Boland CR, et al. Interleukin-6 promotes tumorigenesis by altering DNA methylation in oral cancer cells. Int $J$ Cancer. 2011;129(5):1053-1063.

16. Griffin H, Soneji Y, Van Baars R, et al. Stratification oh HPV-induced Cervical Pathology using the virally-encoded molecular marker E4 in combination with p16 or MCM. Mod Pathol. 2015;28(7):977-993.

17. Guerra E, Paula E. Immunohistochemical expression of cyclin D1 and p16 in oral squamous cell carcinoma: correlation with TNM system and localization; Cyclin D1 and p16. Revista Brasileira de Cancerologia. 2005;51(1):31-37.

18. Hiesch R. Expression of p16 protein, cyclin D1, CDK4 and retinoblastoma proteins in acral lentiginous melanoma. 2008. $99 \mathrm{f}$ Thesis (Master of Science). São Paulo: School of Medicine of the City of São Paulo; 2008.

19. Ledeboer QC, van der Schroeff MP, Pruyn JF, et al. Survival of patients with palliative head and neck cancer. Head Neck. 2011;33(7):10211026.

20. Lokker ME, Offerman MP, van der Velden LA, et al. Symptoms of patients with incurable head and neck cancer : Prevalence and impact on daily functioning. Head Neck. 2013;35(6):868-876.

21. Osazuwa-Peters N, Adjei Boakye E, Chen BY, et al. Sociodemographic factors associated with knowledge and risk perception of human papillomavirus and human papillomavirus-associated oropharyngeal squamous cell carcinoma among a predominantly black population. JAMA Otolaryngol Head Neck Surg. 2017;143(2):117-124.

22. Majchrzak E, Szybiak B, Wegner A, et al. Oral cavity and oropharyngeal squamous cell carcinoma in young adults: a review of the literature. Radiol Oncol. 2014;48(1):1-10.

23. Melkane AE, Mirghani H, Aupérin A, et al. ScienceDirect HPV-related oropharyngeal squamous cell carcinomas : A comparison between three diagnostic approaches. Am J Otolaryngol. 2014;35(1):25-32.

24. Miyahara GI, Simonato LE, Mattar NJ, et al. Correlation between koilocytes and human papillomavirus detection by PCR in oral and oropharynx squamous cell carcinoma biopsies. Mem Inst Oswaldo Cruz. 2011;106(2):166-169. 
25. Queiroz ABP, Focchi GRA, Gomes TS, et al. Study of p27, p21, p16 in normal squamous epithelium, squamous papilloma and oral cavity squamous cell carcinoma. J Bras Patol Med Lab Rio de Janeiro. 2009;45(6):481-488.

26. Quintero K, Giraldo GA, Uribe ML, et al. Human papillomavirus types in cases of squamous cell carcinoma of head and neck in Colombia. Braz J Otorhinolaryngol. 2013;79(3):375-381.

27. Ramqvist T, Dalianis T. Oropharyngeal cancer epidermic and human papillomavirus. Emerg Infect Dis. 2010;16(11):1671-1677.

28. Robinson M, Schache A, Sloan P, et al. HPV specific testing: a requirement for oropharyngeal squamous cell carcinoma patients. Head Neck Pathol. 2012;6 Suppl 1:S83-S90.

29. Vokes EE. Head and neck cancers. In: Long DL. Harrison internal medicine. 18th ed. Porto Alegre: AMGH; 2013.
30. Wittekindt C, Klussmann JP. Tumor staging and HPV-related oropharyngeal cancer. Recent Results Cancer Res. 2017;206:123-133.

31. White EA, Münger K, Howley PM. High-risk human papillomavirus E7 proteins target PTPN14 for degradation. Journal of the American Society for Microbiology. 2016;7(5):1-11.

32. Young RJ, Rischin D, Fisher R, et al. Relationship between epidermal growth factor receptor status, p16 INK4A, and outcome in head and neck squamous cell carcinoma. Cancer Epidemiol Biomarkers Prev. 2011;20(6):1230-1237.

33. Zaravinos A. An updated overview of HPV-associated head and neck carcinomas. Oncotarget. 2014;5(12):3956-3969. 